\title{
Neurobiological considerations in understanding behavioral treatments for pathological gambling
}

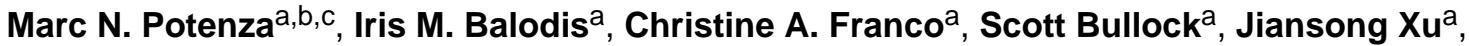 \\ Tammy Chung ${ }^{d}$, and Jon E. Grant ${ }^{e}$ \\ aDepartment of Psychiatry, Yale University School of Medicine, New Haven, Connecticut \\ bDepartment of Neurobiology, Yale University School of Medicine, New Haven, Connecticut \\ ${ }^{\circ}$ Child Study Center, Yale University School of Medicine, New Haven, Connecticut \\ dDepartment of Psychiatry, University of Pittsburgh Medical Center, Pittsburgh, Pennsylvania \\ eDepartment of Psychiatry, University of Minnesota School of Medicine, Minneapolis, Minnesota
}

\begin{abstract}
Pathological gambling (PG), a disorder currently categorized as an impulse-control disorder but being considered as a non-substance addiction in DSM-5 discussions, represents a significant public health concern. Over the past decade, considerable advances have been made with respect to understanding the biological underpinnings of PG. Research has also demonstrated the efficacies of multiple treatments, particularly behavioral therapies, for treating PG. Despite these advances, relatively little is known regarding how biological measures, particularly those assessing brain function, relate to treatments for PG. In this article, we present a conceptual review focusing on the neurobiology of behavioral therapies for PG. To illustrate issues related to study design, we present proof-of-concept preliminary data that link Stroop-related brain activations prior to treatment onset to treatment outcome in individuals with PG receiving a cognitive behavioral treatment incorporating aspects of imaginal desensitization and motivational interviewing. We conclude with recommendations about current and future directions regarding how to incorporate and translate biological findings into improved therapies for individuals with non-substance and substance addictions.
\end{abstract}

\section{Keywords}

Gambling; neuroimaging; treatment development; behavioral therapy; cognitive control; nutraceuticals

Correspondence concerning this article should be addressed to Marc N. Potenza, MD, PhD, Yale University School of Medicine, CMHC, 34 Park Street, New Haven, CT, 06519, USA. marc.potenza@yale.edu..

Disclosures: The authors report that they have no financial conflicts of interest with respect to the content of this manuscript. Dr. Potenza has received financial support or compensation for the following: Dr. Potenza has consulted for and advised Boehringer Ingelheim; has consulted for and has financial interests in Somaxon; has received research support from the National Institutes of Health, Veteran's Administration, Mohegan Sun Casino, the National Center for Responsible Gaming and its affiliated Institute for Research on Gambling Disorders, and Forest Laboratories, Ortho-McNeil, Oy-Control/Biotie, Psyadon and Glaxo-SmithKline pharmaceuticals; has participated in surveys, mailings or telephone consultations related to drug addiction, impulse control disorders or other health topics; has consulted for law offices and the federal public defender's office in issues related to impulse control disorders; provides clinical care in the Connecticut Department of Mental Health and Addiction Services Problem Gambling Services Program; has performed grant reviews for the National Institutes of Health and other agencies; has guest-edited journal sections; has given academic lectures in grand rounds, CME events and other clinical or scientific venues; and has generated books or book chapters for publishers of mental health texts. 
Pathological gambling (PG) has been estimated to affect $0.2 \%-2 \%$ of the general adult population, with several-fold higher estimates reported in adolescents and young adults (Brezing, Derevensky, \& Potenza, 2010; Desai \& Potenza, 2008; Petry, Stinson, \& Grant, 2005; Shaffer, 1999). Despite its considerable prevalence and the significant personal, familial and societal costs associated with PG (Gerstein et al., 1999; Potenza, Kosten, \& Rounsaville, 2001), relatively little research has been conducted into its etiology and treatment. An improved knowledge of clinical and biological processes, combined with a better understanding of treatment mechanisms is important for developing improved and more targeted treatments in PG and addictions in general (Potenza, Sofuoglu, Carroll, \& Rounsaville, 2011).

In this article, we explore the neurobiological mechanisms of behavioral therapies for PG. We first describe a current understanding of the neurobiology of PG. Next, we review the empirical support for behavioral interventions in PG (reviewed elsewhere in greater detail (Brewer, Grant \& Potenza, 2008; Stea \& Hodgins, 2011)), with a description of how these therapies might function at a neurobiological level. Then, to illustrate the approaches described, we present proof-of-concept data linking fMRI (functional magnetic resonance imaging) data to treatment outcome in PG patients receiving behavioral therapy. We conclude with thoughts about future directions in treatment development for PG.

\section{Neurobiology of Pathological Gambling: Implications for Treatment Development}

Over the past decade, considerable advances have been made in understanding the biological contributions to PG and how they are similar to and distinct from those underlying substance use disorders (as reviewed in (Frascella, Potenza, Brown, \& Childress, 2010; Leeman \& Potenza, 2012; Potenza, 2006; Potenza, 2008)). Similarities in neurobiological, genetic, clinical and phenomenological features between PG and substance dependence is prompting consideration of movement from the category of "Impulse Control Disorders Not Elsewhere Classified" to one including both substance and non-substance addictions (American Psychiatric Association Committee on Nomenclature and Statistics, 2000; Holden, 2001, 2010). As this reclassification has considerable implications for how one might conceptualize and approach PG in research, prevention, treatment and policy domains, a brief review of some main findings is warranted.

Similarities between PG and substance-use disorders include clinically relevant features that might be targeted in behavioral therapies, and these include urges/cravings, disadvantageous decision-making and poor impulse control. Several aspects that may be targeted in behavioral therapies may hold greater relevance in PG. For example, financial problems may be more severe and financial management thus more relevant. Cognitive distortions may also be particularly salient for PG, with near-misses (e.g., when the first two reels of a slot machine displays matching symbols and then the third does not) representing one example. Additionally, loss-chasing (going back to a venue of gambling to try to win back money recently lost) is reflected in the diagnostic criteria for PG but not for substance-use disorders. These states and processes (urges/cravings, decision-making, near-miss processing and loss-chasing) each engage different aspects of brain circuitry, particularly those involved in motivation, executive control and reward/loss processing. In some cases, PG and substance-use disorders have been compared and contrasted, with both similarities and differences observed (reviewed in Leeman \& Potenza, 2012).

With respect to biological similarities between PG and substance-use disorders, a particularly strong relationship might exist with alcohol dependence, although similarities also appear to extend to other addictions like nicotine and cocaine dependence (Grant, 
Black, Stein, \& Potenza, 2009; Potenza, 2008). Shared genetic contributions have been reported between PG and alcohol dependence (Slutske et al., 2000), and such shared factors might be reflected in performance on cognitive tasks involving risk/reward decision-making as well as responses to specific pharmacological treatments like opioid antagonists and glutamatergic agents like n-acetyl cysteine (described in greater detail in supplementary materials) (Lawrence, Luty, Bogdan, Sahakian, \& Clark, 2009; Potenza, 2009).

Consistent with observations that opioidergic and glutamatergic drugs may exert therapeutic benefits across non-substance and substance addictions, similar contrasts between individuals with PG and comparison subjects have been found in neuroimaging studies of reward processing as have been found between alcohol-dependent and comparison subjects on a similar task (Balodis et al., 2012; Beck et al., 2009; Wrase et al., 2007). Specifically, individuals with alcohol dependence have shown relatively diminished activation in the VS during the processing of monetary rewards, particularly during the anticipatory phase, and similar results have recently been observed in individuals with PG. These findings might relate to specific neurotransmitter systems in the VS, as VS dopamine receptor function has been implicated in PG (Steeves et al., 2009). However, as other groups have not identified PG-related between-group differences in VS dopamine (Linnett et al., 2010, 2011; Clark et al., 2012), VS differences related to other neurotransmitters also warrant consideration. For example, serotonin 1B receptor function in the VS has been implicated in both PG and alcohol dependence (Hu et al., 2010; M N Potenza et al., in press). Importantly, the relatively diminished ventral striatal activation during reward anticipation in PG has been linked to impulsivity (Balodis et al., 2012; Beck et al., 2009). Thus, behavioral therapies targeting improved impulse control as related to reward processing in PG might lead to changes in this region during effective treatment.

Other brain regions have also been frequently implicated in PG. For example, relatively diminished activation of the ventromedial prefrontal cortex (vmPFC) has been observed in PG across several studies including ones involving reward processing, gambling urges and simulated gambling (Balodis et al., 2012; Potenza, Steinberg, et al., 2003; Reuter et al., 2005). Relatively diminished vmPFC activation has also been observed in PG during other tasks assessing domains distinct from reward processing (Potenza, Leung, et al., 2003; Tanabe et al., 2007). For example, individuals with PG have shown relatively diminished activation of the vmPFC during a cognitive control task, the Stroop color-word interference test (Potenza, Leung, et al., 2003).

\section{The Efficacy of Behavioral Treatment of PG}

Multiple behavioral therapies for PG have been developed based on those showing efficacy for substance-use disorders, although some approaches that have demonstrated efficacy to varying degrees (e.g., contingency management, harm reduction) have not been systematically investigated in the treatment of PG. Therapies targeting erroneous cognitions, gambling urges and motivations appear efficacious in the treatment of PG. A meta-analysis of behavioral therapies in PG collapsed across therapy types (Pallesen, Mitsem, Kvale, Johnsen, \& Molde, 2005) indicated a large effect size of 2.01 at the end of treatment and an effect size of 1.59 upon follow-up (average of 17 months), suggesting a significant albeit reduced impact over time. These effect sizes may be considered large, taking into account Cohen's classification of large effect sizes being those of 0.8 or greater. 


\section{Using Neuroimaging to Examine Mechanisms Underlying Behavioral Interventions for PG}

Brain imaging offers the opportunity to investigate the mechanisms underlying effective behavioral therapies for PG. As each behavioral therapy may operate through distinct active ingredients, specific cognitive tasks may be optimal for understanding how behavioral therapies mediate their therapeutic effects in PG. Given fMRI's combination of temporal and spatial resolution and the relative ease of incorporating cognitive tasks, fMRI appears well suited to understand the neural mechanisms by which specific behavioral therapies operate (see summarized information in Table 1). Cognitive tasks that link directly to theoretically postulated mechanisms of action of behavioral therapies or their components may be performed during fMRI and shed insight into how brain activations (both regional and in functional networks) may relate to specific treatments as well as to individual differences in treatment responses (e.g., normalization of brain circuitry or compensatory processes associated with individual differences in the integrity of brain structure and function). The proposed fMRI tasks could be used pre-treatment, during treatment or after treatment to investigate how baseline brain function relates to treatment outcome and/or how brain function changes during the course of treatment.

A description follows of specific therapies for PG (both those with empirical support and those with potential promise), their proposed active ingredients, and fMRI tasks that may be well suited to investigate the neural mechanisms underlying their efficacies. Specifically, the following sections discuss some of the possible "active ingredients" for Cognitive Behavioral Therapy, Aversive Therapies, Imaginal Desensitization/Exposure Therapy, Motivational Interviewing, Gamblers Anonymous, Mindfulness-Based Stress Reduction and Meditation, and Contingency Management (Table 1). The interventions may have "common" effects in addressing brain regions and circuits involved in motivation (e.g., cortico-striatal-pallido-thalamo-cortical circuitry), executive control (e.g., dorsal lateral prefrontal cortex and anterior cingulate cortex), and reward/loss processing (e.g., ventral striatum, vmPFC and insula). In addition, "intervention-specific" effects (e.g., mindfulnessbased intervention effects in optimizing default mode functioning (e.g., in vmPFC and posterior cingulate) or decreasing stress responsivity (e.g., in amygdala, hippocampus, striatum, insula and cortical regions)) might be observed. The hypothesized active ingredients of these interventions are discussed in relation to predicted effects on neurocircuitry, and in relation to specific fMRI tasks (including example studies using the fMRI tasks) that could be used to examine the proposed mechanism of treatment effects. Given the multi-component nature of many behavioral treatments, it remains challenging to isolate effects if a particular active ingredient based on a single fMRI task. In this context, the following sections provide a heuristic framework for how fMRI can be used to examine mechanisms of treatment change for behavioral interventions.

\section{Cognitive Behavioral Therapy (CBT)}

In different versions of cognitive behavioral therapy (CBT) adapted for PG (Ladouceur \& Lachance, 2007a, 2007b; Petry, 2005b; Sylvain, Ladouceur, \& Boisvert, 1997), individuals are taught to use functional analyses (i.e., identifying triggers, related feelings, resulting behaviors, and describing positive and negative consequences) to examine the positive and negative consequences of their triggers and to identify cognitive biases (e.g., luck-related beliefs) for their gambling behaviors. The former aspect of developing improved strategies to identify and manage triggers related to craving may function through increasing prefrontal cortical control over motivational drives involving subcortical brain regions (Potenza et al, 2011). The latter aspect might involve increased awareness and a better understanding of irrational cognitions (e.g., relating to luck, near-miss events (losses that 
may be considered "near wins"), and chasing behaviors) that may involve balancing activity of brain circuits coding conflicting motivational states (e.g., increased activation of dorsal anterior cingulate, insula and prefrontal cortex relative to reward/motivational systems).

CBT for PG may include modules addressing the performance of functional analyses, planning and decision-making focused on making healthier choices and engaging in nonaddictive pleasant activities, coping with urges to gamble, assertiveness learning and gambling refusal (managing conflicting choices, like those identified in functional analyses), changing irrational or biased beliefs, and improving financial management (Petry, 2005b). Specific fMRI tasks might link directly or theoretically to these areas of skill development. For example, gambling-cue-exposure tasks might be particularly relevant to understanding changes related to coping with gambling urges (Crockford, Goodyear, Edwards, Quickfall, \& El-Guebaly, 2005; Potenza, Steinberg, et al., 2003), and fMRI tasks that involve attempts to regulate these motivational states may also be particularly relevant (Kober et al., 2010). Treatment-related changes in financial management skills, which might involve altered processing of immediate versus delayed rewards (and brain circuitry related to reward processing and decision-making), might be assayed with fMRI tasks that involve monetary reward processing and delay discounting (Balodis et al., 2012; Miedl, Peters, \& Buchel, 2012). CBT-related improvement in managing conflicts might be examined with fMRI decision-making and cognitive control tasks might be particularly relevant to understanding changes related to managing conflicts (Potenza, Leung, et al., 2003; Tanabe et al., 2007). "Near-miss" tasks might be particularly relevant to understanding changes related to cognitive biases (Chase \& Clark, 2010; Clark, Lawrence, Astley-Jones, \& Gray, 2010; Habib \& Dixon, 2010). In particular, the last set of tasks might detect an important role for the dorsal anterior cingulate, a region also implicated in cognitive control processes among PG subjects (Potenza, Chung, et al. 2003).

CBT also may involve teaching (through role-playing and skills training) interpersonal skills and conflict resolution methods. These skills are meant to help the person recognize that gambling, although linked to short-term enjoyment, has long-term consequences that are problematic - such as heavy debt, trouble with family/friends or the legal system. This type of intervention further addresses finance management and debt settlement - important concerns in PG (Petry, 2005a). CBT-related changes in conflict resolution (e.g., decisions between short-term enjoyment versus longer-term consequences) might involve changes in decision-making, risk-taking and reward processing that are mediated by prefrontal, striatal and limbic brain regions. Changes in these essential elements may best be captured through the use of specific fMRI tasks assessing decision-making (e.g., the Iowa Gambling or InterTemporal Choice Task), risk-taking (e.g., the Balloon Analog Risk Task) or reward processing (e.g., the Monetary Incentive Delay Task).

CBT also has the advantage in that a trained clinician can deliver the therapy in either individual or group format. In addition, CBT is effective in conjunction with pharmacotherapy and appears efficacious for individuals with co-occurring disorders (Champine \& Petry, 2010; Petry, 2005a; Toneatto, Brands, \& Selby, 2009). Importantly, improvement associated with this therapy appears long-lasting; a meta-analysis of CBT in PG indicated significant gains at end of treatment (three months) as well as at six-, twelveand twenty-four-month follow-up time points (Gooding \& Tarrier, 2009). These findings are consistent with those from the drug addiction literature in which CBT as compared to other behavioral therapies appears particularly robust over time, perhaps through the continued use and developing expertise of CBT-related skills (Carroll, Rounsaville, Nich, Gordon, Wirtz, \& Gawin, 1994; Carroll et al., 2009). Understanding the neural underpinnings of such delayed or persistent responses is of significant clinical relevance. 


\section{Aversive and Imaginal Therapies (Exposure Therapies)}

Aversive and imaginal therapies may be considered as forms or components of CBT or as exposure therapies. Aversive therapies are based on classical conditioning (respondent conditioning) theory. Treatment methods include aversion therapy (reduces frequency of a behavior by repeated pairings with negative, unpleasant stimuli), imaginal desensitization (the subject is guided through imaginal experiences of gambling and taught how to control their physiological responses so that in future cases of exposure to gambling cues, they can better control their behavior and avoid gambling), and in-vivo exposure (being exposed to gambling in a real-life setting in order to become desensitized and develop different habits). Such training may alter habitual responding, perhaps through alteration of functioning of habit-based circuitry involving the orbitofrontal cortex and dorsal striatum (Hampshire \& Owen, 2006; Rogers, Andrews, Grasby, Brooks, \& Robbins, 2000; Yin \& Knowlton, 2006). FMRI tasks assessing habitual responding and cognitive flexibility (e.g., the intradimensional/extra-dimensional set-shifting task) or those assessing cognitive control (e.g., the Stroop color-word interference task) may be particularly relevant to understanding the efficacies of these approaches (see proof-of-concept section below and Table 1). Varying degrees of success in treating PG and other impulse-control disorders with these behavioral interventions have been reported (Hodgins \& Peden, 2008; Tavares, 2003). Studies have compared imaginal desensitization to aversive therapy, imaginal relaxation, and in vivo exposure. Those receiving imaginal desensitization fared better in long-term reductions in gambling behavior compared to the other forms of treatment (McConaghy, Blaszczynski, \& Frankova, 1991).

\section{Motivational Therapies and Brief Interventions}

Motivational interviewing and adaptations thereof (motivational enhancement therapy and brief motivational interviewing) have been well-validated in the treatment of substance addictions (Miller, 1996; Miller, Yahne, \& Tonigan, 2003). Motivational interviewing has been recently defined as, "a collaborative, person-centered form of guiding to elicit motivation for change" (Miller \& Rollnick, 2009). Motivational techniques often involve rolling with a patient's resistance and exploring with the patient in an unbiased fashion the pros and cons of specific behaviors. Motivational therapies in PG may operate through dampening of reward/motivational circuitry associated with gambling, and change-talk combined with fMRI cue-reactivity tasks might be particularly relevant to understanding motivational interventions (Feldstein Ewing, Filbey, Hendershot, McEachern, \& Hutchison, 2011) (Table 1).

Brief interventions (often lasting under 10 minutes and fewer than four sessions) have been widely used in behavioral disorders and may include elements leading to motivational enhancement. The contents of brief interventions vary and can be as simple as a brochure detailing the effects a specific behavior can have on you or those around you. Although the active ingredient of brief interventions remains speculative, brief interventions may have an effect in catalyzing motivation, similar to MI/MET (i.e., through having individuals weigh the pros and cons of specific behaviors and alter decision-making processes based on these reflections).

In a randomized trial of MET/CBT and brief interventions for PG (Petry, Weinstock, Ledgerwood, \& Morasco, 2008), subjects were randomly assigned to one of four conditions: assessment only (control), brief advice (an approach involving some aspects of motivational interviewing such as individualized personal feedback), motivational enhancement therapy (MET - encouraging individuals to identify pros and cons of altering gambling behavior along with providing feedback), or MET combined with CBT. The brief advice condition 
was the only one demonstrating significant improvement (as determined by South Oaks Gambling Screen [SOGS] scores and dollars wagered) by week six, with gains evident also at nine months. Other interventions (e.g., CBT and MET), showed more delayed improvements. These results suggest that brief advice incorporating some aspects of motivational interviewing may be helpful for non-treatment-seeking individuals with problem and PG.

\section{Gamblers Anonymous}

The most widely used intervention for PG is Gamblers Anonymous (GA), an approach modeled on Alcoholics Anonymous. Like Alcoholics Anonymous, GA is not a behavioral therapy, although many of the potential active ingredients (e.g., social support for abstinence) involved in GA meetings may influence gambling behaviors, particularly those related to relapse prevention. As is the case with the behavioral therapies described above, the precise components underlying GA's efficacy may be multiple and difficult to disentangle. For example, aspects involving admitting being "powerless" over addictive behaviors (which then might permit greater control over the addictive behaviors through changes in decision-making processes surrounding gambling) may contribute. Additionally, social interactions may reflect an active ingredient that may have effects through being in contact with similarly motivated peers sharing a goal of abstinence (e.g., a social norm of abstinence), and access to resources (e.g., sponsors) in developing skills for managing crises. Specific aspects of GA may thus operate through different mechanisms; for example, influences on decision-making and risk-taking behaviors might be encountered in GA meetings or in discussions with sponsors. Thus, the effective components of GA might be understood through specific fMRI tasks investigating social influences on decision-making and risk-taking behaviors (Cavalca et al., 2012; Hyatt et al., 2012; Rao et al., 2010; Tanabe et al., 2007). Existing data suggest the involvement of brain regions implicated in moral or pro-social processes (including the orbitofrontal cortex (Leeman, Grant, \& Potenza, 2009)), and changes in decision-making and risk-taking behaviors might involve the ventromedial prefrontal cortex and ventral striatum, respectively (Bechara, 2003; Tanabe et al., 2007; Rao et al, 2010) (Table 1). Although there are hundreds of GA chapters worldwide, attrition rates in GA appear high (Stewart \& Brown, 1988), making the efficacy of GA hard to determine. Studies investigating GA attendance in association with professional treatment have found that abstinence from gambling is higher for those engaged in both modalities (Petry, 2003).

\section{Other Behavioral Therapies}

Other fMRI tasks might be particularly well suited for understanding other purported mechanisms of behavioral therapies that currently have less empirical support in PG; for example, stress or cue-exposure tasks may be particularly relevant for understanding the mechanisms underlying mindfulness-based stress reduction (Brewer, Smith, Bowen, Marlatt, \& Potenza, 2010; Potenza et al., 2012), meditation-based fMRI tasks (e.g., imaging during active meditation states) may be particularly relevant for understanding the mechanisms underlying specific forms of meditation (Brewer et al., 2011), and reward processing and inter-temporal choice tasks for contingency management (Balodis et al., 2012; Miedl et al, 2012).

\section{Combination Therapies - Imaginal Desensitization and Motivational Interviewing}

As mentioned above, individual therapies may work through multiple individual mechanisms, and combinations of specific therapies that operate in the manners proposed above may act synergistically. In a study comparing CBT with a focus on imaginal 
desensitization and motivational interviewing (IDMI) to Gambler's Anonymous (GA), 68 individuals with PG were assigned to receive either six sessions of IDMI over an eight-week period, or attend GA for the same amount of time (Grant, Donahue, Odlaug, \& Kim, 2011; Grant, Donahue, et al., 2009). Those not responding to GA were given the six sessions of IDMI after the eight-week acute treatment period. In all, 44 participants completed six sessions of IDMI (19 from the original GA group who did not respond to GA) with 35 defined as responders (35\% or greater reduction in Yale Brown Obsessive Compulsive Scale adapted for PG (PG-YBOCS) scores), representing a 79.55\% response rate amongst completers. At a six-month follow-up, all scales still showed significant improvement from baseline scores, with 27 individuals remaining abstinent. This approach may be also applicable to a broader range of impulse control disorders (Grant, Donahue, \& Odlaug, 2011a, 2011b).

\section{Summary of the Use of fMRI to Examine Effects of Behavioral Interventions for $P G$}

As each of the fMRI tasks listed above might differentially engage specific brain regions and networks, and do so to different degrees in different people, the integration of these tasks into clinical trials examining the efficacies of behavioral therapies offers significant opportunities for understanding the neural functions underlying the effective ingredients of behavioral therapies and related processes. An improved understanding of how individual differences in brain function underlie clinical characteristics and relate to treatment outcome may ultimately help in the currently elusive process of effectively matching treatmentseeking individuals with addictions to specific therapies. Ideally, each task would be performed pre- and post-treatment, with consideration of and attention to possible habituation or learning effects.

\section{Relating Brain Activations to Treatment Outcome in PG}

Relatively few studies have examined how neuroimaging measures relate to treatment outcome in PG. One report used functional magnetic resonance imaging (fMRI) in a single subject to explore regional activations associated with fluvoxamine treatment (Chung et al., 2009). This study found that in this subject, frontal, parietal and occipital activations to the presentations of playing cards (e.g., showing a full house, four of a kind) was observed at baseline, with similar contrasts not showing such extensive activations over time. However, the findings from this study should be considered cautiously given the small sample $(n=1)$ and the lack of a control group to examine the extent to which any changes might be related to specific aspects of treatment (e.g., the fluvoxamine) versus test-retest changes independent of treatment, baseline problem gambling severity, or changes therein. A second study using positron emission tomography (PET) examined differences between 21 individuals with PG and bipolar-spectrum disorders (largely bipolar II disorder) and 21 nonaffected control subjects and found that the PG/bipolar subjects had lower regional glucose metabolic rates in subcortical regions including the VS, and that lithium treatment (associated with better treatment outcome as compared to placebo (Hollander, Pallanti, Allen, Sood, \& Rossi, 2005)) was associated with increasing the regional glucose metabolic rate in the VS (Pallanti et al., 2010). Together, these findings suggest that VS activity may relate to baseline problem gambling status or severity and that treatment might "normalize" function in this region and thus reduce problem gambling behaviors. However, the extent to which this might hold true for non-bipolar PG subjects has not been examined. 


\section{Stroop fMRI Task Performance as a Predictor of Treatment Outcome}

Performance on the Stroop has been associated with treatment outcomes for substance addictions like cocaine dependence (Streeter et al., 2007), and the brain correlates of Stroop performance (assessed using fMRI) have been associated with treatment outcome in cocaine dependence (Brewer, Worhunsky, Carroll, Rounsaville, \& Potenza, 2008). Specifically, Stroop-related activation of the vmPFC and striatum at treatment onset were associated with better treatment outcome (as assessed by self-reported and urine-toxicology-based drug abstinence) amongst cocaine-dependent patients receiving behavioral therapies (Brewer, Worhunsky, et al., 2008). Together with the data highlighting similarities between PG and substance addictions and those from studies of the neural underpinnings of PG presented above, the findings provide a strong rationale for directly investigating the relationship between the brain activations during Stroop performance (specifically in the vmPFC and striatum) and treatment outcome in PG. As described below (see the following section on proof-of-concept pilot data), the Stroop task may be particularly relevant to understanding the mechanisms of specific behavioral therapies for PG, particularly those which involve the generation or acquisition of greater self-control over gambling behaviors.

\section{Proof-of-Concept Preliminary Data}

To demonstrate the potential of using brain imaging to explore the relationship between the fMRI correlates of cognitive control and treatment outcome in PG (particularly as related to the proposed active ingredient of IDMI - controlled exposure to gambling cues that may increase prefrontal control over motivational systems), we obtained fMRI Stroop measures prior to treatment onset in individuals with PG seeking treatment for their gambling problems. Each subject agreed to participate in a study in which they received six weeks of a CBT involving IDMI therapy, as well as receiving in a placebo-controlled fashion the amino-acid dietary supplement $\mathrm{N}$-acetyl cysteine, a nutraceutical that through glutamatergic mechanisms can influence VS dopaminergic function (additional methodology included in supplemental materials). Based on data indicating inverse correlations between vmPFC and VS activation during simulated gambling and problem-gambling severity in PG (Reuter et al., 2005), we hypothesized (hypothesis 1) that vmPFC and VS activation during Stroop performance would correlate inversely with pre-treatment problem-gambling severity as assessed by the Yale-Brown Obsessive-Compulsive Scale adapted for Pathological Gambling (PG-YBOCS). Based on findings in which pre-treatment activation during fMRI Stroop was positively correlated with better treatment outcome in cocaine dependence (J. Brewer et al., 2008), we hypothesized (hypothesis 2) that vmPFC and VS activation during fMRI Stroop prior to treatment onset would correlate with improvement during treatment as assessed by changes in PG-YBOCS scores.

FMRI data were collected using previously published methodologies (J. Brewer et al., 2008) and are described in detail in the supplementary materials. Participants were 7 treatmentseeking individuals ( 2 females) with ages ranging from 41-56 years of age who met criteria for PG and nicotine dependence, were seeking help for their gambling problems and participated in a double-blind, randomized, placebo-controlled trial of $\mathrm{N}$-acetyl cysteine (see ClinicalTrials.gov [www.clinicaltrials.gov] with registry identifier NCT00967005). The trial included twelve weeks of treatment with $\mathrm{N}$-acetyl cysteine or placebo and six weeks of CBT with imaginal desensitization motivational interviewing (IDMI) as described elsewhere (Grant, Donahue, et al., 2009). IDMI therapy was initiated at week 7 of the trial. As the main findings from the trial are currently unpublished, data blind to treatment condition are presented. All subjects provided written informed consent for research approved by the Yale University Human Investigations Committee. 
The PG-YBOCS, a valid, reliable 10-item clinician-administered questionnaire developed to measure the severity and change of PG symptoms, assessed baseline (pre-treatment) and changes in problem-gambling severity (Pallanti, DeCaria, Grant, Urpe, \& Hollander, 2005). Whole-brain maps contrasting neural activations underlying incongruent versus congruent stimuli identified brain regions previously implicated in Stroop performance including the dorsal anterior cingulate, dorsolateral prefrontal cortex, insula, striatum and thalamus (Supplemental Figure 1). Whole-brain correlations between baseline PG-YBOCS scores and Stroop performance largely identified inverse correlations (Supplemental Table 2;

Supplemental Figure 2). Regions identified included the dorsal anterior cingulate cortex (extending into perigenual and ventral anterior cingulate), midbrain, amygdala,

hippocampus, striatum, insula, thalamus, lateral portions of ventral prefrontal cortex, thalamus, parietal cortex, occipital cortex and cerebellum. Of the regions hypothesized to correlate with problem-gambling severity, bilateral ventral striatal activation correlated inversely with problem-gambling severity (Figure 1a; Supplemental Table 2). The vmPFC was largely not identified (with the exception of its more dorsal component on the periphery of the activation involving the anterior cingulate; Supplemental Figure 2).

Whole-brain correlations were performed between PG-YBOCS change scores (12-week versus baseline) and regional brain activity during Stroop performance, contrasting incongruent versus congruent trials (Figure 1b, c; Supplemental Table 3; Supplemental Figure 3). Changes in PG-YBOCS scores correlated positively with activation in the vmPFC including in the orbitofrontal cortex and medial frontal gyrus (Figure 1b) and the right ventral striatum including the nucleus accumbens (Figure 1c). Activity in additional brain regions, including the amygdala, hippocampus, parahippocampus, inferior temporal gyrus and occipital cortex, also correlated with changes in PG-YBOCS scores (Supplemental Table 3; Supplemental Figure 3). In all cases, correlations showed that lower activity during baseline scanning were associated with more improvement as indicated by changes in scores on the PG-YBOCS.

Taken together, the presented preliminary findings linking pre-treatment brain activations to baseline problem-gambling severity and reductions in problem-gambling severity during treatment with a CBT involving IDMI (and either placebo or $\mathrm{N}$-acetyl cysteine) suggest important roles for the VS and vmPFC in treatment outcome for PG. The findings resonate with prior fMRI Stroop studies in substance dependence linking VS and vmPFC activation to drug abstinence (Brewer et al., 2008; Worhunsky et al. in press) and a prior PET study linking baseline VS function and changes in VS function during lithium treatment in individuals with PG and bipolar-spectrum disorders (Pallanti et al., 2010).

The implications of these preliminary findings are discussed in greater detail in the supplementary materials. While it is tempting to speculate that the activations (particularly in the vmPFC) might relate to the specific actions of the IDMI therapy (e.g., to involvement of the vmPFC as related to risk-reward decision-making (Bechara, 2003)), one should be cautious about such interpretations for several reasons. First, the sample size in this study is very small. Second, the VS and vmPFC have been found to work in conjunction with one another. For example, both regions have been implicated in reward processing (Knutson, Fong, Bennett, Adams, \& Hommer, 2003), activate during the selection of smaller, immediate versus larger delayed rewards (McClure, Laibson, Loewenstein, \& Cohen, 2004), and demonstrate activity during simulated gambling correlating similarly (inversely) with problem-gambling severity in PG (Reuter et al., 2005). Importantly, the vmPFC and VS have been identified in an independent component analysis of fMRI Stroop performance as contributing to a functionally integrated set of brain activations (Worhunsky et al., in press), suggesting that these regions work in concert during Stroop performance. Given that this set 
of activations related to abstinence during treatment of cocaine-dependent individuals, it is tempting to speculate that a similar process might underlie effective treatment in PG.

The extent to which changes in problem-gambling severity might relate to the behavioral therapy employed (a CBT involving IDMI) warrants further investigation. It is tempting to speculate that the greater reductions in problem-gambling severity might relate to behavioral-therapy components received or performed. The neural underpinnings of MI in the treatment of alcohol dependence have been studied with preliminary data suggesting that change talk in MI may alter alcohol-cue-related activations in regions including the VS and vmPFC (Feldstein Ewing, Filbey, Sabbineni, Chandler, \& Hutchison, 2011). Based on these findings, a model involving emotional/learning/memory, incentive reward and executive control circuits has been proposed for MI in alcohol dependence (Feldstein Ewing, Filbey, Hendershot, et al., 2011), and the extent to which such a model might apply to PG warrants direct investigation.

These proof-of-concept findings build upon prior studies exploring the neurobiology of treatment outcome in PG. As compared to the sole previously published fMRI report (Chung et al., 2009), this study employed a larger sample and arguably better defined treatment conditions. Nonetheless, the small sample in the present study is a limitation and represents one factor restricting the ability to relate the findings to specific treatments or aspects thereof. Other limitations of this proof-of-concept study include the absence of a group not receiving behavioral therapy, mixture of behavioral therapies employed, one participant not receiving behavioral therapy, lack of all possibly clinically relevant measures (e.g., of antisociality) and blinded nature of the findings with respect to $\mathrm{N}$-acetyl cysteine administration due to the ongoing nature of the study. In addition, pre-post imaging measures were not examined, but pre-post change in brain activation might be predicted to involve midbrain regions that have demonstrated pre/post-treatment changes beyond test/ retest changes during Stroop performance in substance abuse treatment (DeVito et al., 2012), particularly as gambling severity has been linked to midbrain responses to near-miss events (Chase \& Clark, 2010).

\section{Current Status and Future Directions in Using fMRI to Understand Mechanisms of Behavioral Interventions for PG}

Currently we are at an early stage of understanding the neurobiology of PG and effective behavioral treatments for the disorder, and at a particularly early stage in understanding the relationships between these domains. By integrating neuroimaging measures into clinical trials for behavioral therapies for PG, we may move beyond the brain as a "black box" for mediating effective behavioral change and move towards understanding the effective ingredients of specific therapies, and how individual differences in people seeking treatment for PG might relate to these entities.

PG, like substance addictions, involves urges/cravings, disadvantageous decision-making and poor impulse control. Despite features that are specific to PG (e.g., near-misses, losschasing), non-substance and substance addictions show similar impairments in neural circuitry involved in, for example, motivation, executive control, decision-making, and reward/loss processing. The behavioral interventions reviewed address impairments in these neural circuits; however, no studies have used neuroimaging to examine treatment-related change in brain circuitry for PG. The preliminary proof-of-concept fMRI study presented here is the largest to date to use fMRI as a "predictor" of PG treatment outcome, and no studies to date have examined pre/post changes in brain function with respect to behavioral therapy for PG. 
Conceptual issues remain to be addressed in using fMRI to examine treatment-related change in PG. For example, common versus intervention-specific active ingredients need to be identified (i.e., which distinct intervention components are associated with specific outcomes relative to "common" effects that are not specific to a single intervention). Most behavioral interventions include multiple components, which may have optimal effects when used together, rather than when a component is tested for effects in isolation. Specific therapies, like mindfulness-based stress reduction, warrant additional examination in PG populations, and understanding the extent to which such intervention might operate through re-appraisal (involving cognitive mechanisms) of responses to stress or gambling-related cues or through alterations in responses to the stressors or cues themselves (through habituation processes related to changes in behavioral responses over the longer term) is important. Analogously, understanding the neural mechanisms underlying other forms of delayed treatment effects is also clinically relevant. For example, CBT has been shown in drug addiction to have late-emerging, persistent, and durable effects (Carroll, Rounsaville, Nich, Gordon, Wirtz, \& Gawin, 1994; Carroll et al., 2009), and understanding the neural mechanisms responsible for these effects is important.

Methodological issues in using fMRI to examine mechanisms of treatment change also remain to be addressed. For example, larger studies should utilize specific fMRI tasks that relate not only to neuropsychological features of PG (Clark, 2010), but also to the specific mechanisms of actions of the therapies being tested, as has been suggested for both substance and non-substance addictions (Potenza et al., 2011). The fMRI tasks reviewed in the Table provide only a sample of tasks that might be used to probe specific impairments and to track treatment-related change. Reliability and validity of fMRI tasks need to be established, especially test/re-test reliability of fMRI tasks used in pre/post designs.

Future studies should examine further the relationships between PG treatment outcome and the brain correlates of not only cognitive control but also other processes linked to PG (e.g., responses to monetary rewards and losses or other aspects of gambling). Larger studies should also consider potential confounds (e.g., co-occurring psychiatric disorders and influences of substance use) on brain changes associated with behavioral therapies. Possible endophenotypic constructs (e.g. relating to impulsivity, compulsivity, stress responsivity, or emotional regulation) should also be investigated in this context. Future studies should also examine larger, more diverse samples to investigate brain and treatment outcome measures that might relate to specific subgroups (e.g., based on gender, other addictive behaviors and other co-occurring psychopathology).

Other brain imaging measures associated with treatment outcome in substance addictions also warrant investigation in PG. For example, poorer white matter integrity has been associated with poorer abstinence-based outcome in cocaine dependence (Xu et al., 2010). As PG has been associated with poorer white matter integrity (Yip et al., in press) and both pharmacological and behavioral interventions have been associated with improvements in white matter integrity-although the precise mechanism for this change is not well understood (Harsan et al., 2008; Schlaug, Marchina, \& Norton, 2009; Tang et al., 2010), white matter represents a potential treatment target in PG. Similarly, gray matter differences might relate importantly to PG and its treatment, although initial studies involving small samples have not observed differences in regional brain volumes in PG and non-PG subjects (Joutsa, Saunavaara, Parkkola, Niemela, \& Kaasinen, 2011).

\section{Conclusions}

Behavioral interventions are the most widely used approaches to help people with PG. Despite their large effect sizes, little is known regarding the neural mechanisms underlying 
effective changes with these therapies. The availability of fMRI affords a unique and timely opportunity to investigate the active ingredients of specific behavioral therapies for PG. By understanding the brain mechanisms related to specific therapies, one may finely tune and adapt behavioral therapies to increase efficacy.

Identifying the neural mechanisms underlying specific behavioral therapies (and specific components of these therapies) remains an important endeavor and largely unexamined area not only in PG, but also in addictions in general. Brain imaging has the potential (at least theoretically) to inform clinical decision-making, even though this possibility does not seem to be on the immediate horizon. Although not feasible given current limitations in our understanding of the brain processes underlying addictions and how brain changes relate to changes in addictive behaviors, it may be possible in the future to use imaging to guide clinical decision-making, as is now currently done for other conditions like cancer or stroke. Prior to such clinical uses, there is substantial potential for the acquisition of significant scientific knowledge from endeavors that incorporate imaging measures into clinical trials in a theoretically grounded manner. Such interdisciplinary research would help address important knowledge gaps and has significant potential for reducing further the stigma that still exists for addictions. Most importantly, such efforts should help generate improved treatment approaches for addictions and alleviate suffering amongst those with addictions and others adversely impacted through relationships with these people.

\section{Supplementary Material}

Refer to Web version on PubMed Central for supplementary material.

\section{Acknowledgments}

The authors gratefully acknowledge Monica Solorzano for her help with the study and Hedy Kober for advice on analyses(Habib \& Dixon, 2010).

This study was funded by National Institute on Drug Abuse (NIDA) grants R01 DA019039, R01 DA020908, RC1 DA028279, P20 DA027844, P50 DA09241, the Connecticut Department of Mental Health and Addiction Services, and the National Center for Responsible Gaming's Centers of Excellence in Gambling Research at Yale University and the University of Minnesota. Data for this report were drawn from the clinical trial registered with ClinicalTrials.gov (www.clinicaltrials.gov) with registry identifier NCT00967005. We thank the reviewers for their helpful comments.

\section{References}

American Psychiatric Association Committee on Nomenclature and Statistics. Diagnostic and Statistical Manual of Mental Disorders. 4th Ed. - Text Revision. American Psychiatric Association; Washington, DC: 2000.

Balodis IM, Kober H, Worhunsky PD, Stevens MC, Pearlson GD, Potenza MN. Diminished frontostriatal activity during processing of monetary rewards and losses in pathological gambling. Biological Psychiatry. 2012; 71:749-757. [PubMed: 22336565]

Bechara A. Risky business: Emotion, decision-making, and addiction. Journal of Gambling Studies. 2003; 19:23-51. [PubMed: 12635539]

Beck A, Schlagenhauf F, Wustenberg T, Hein J, Kienast T, Kahnt T, Wrase J. Ventral striatal activation during reward anticipation correlates with impulsivity in alcoholics. Biological Psychiatry. 2009; 66:734-742. [PubMed: 19560123]

Brewer JA, Grant JE, Potenza MN. The treatment of pathological gambling. Addictive Disorders and Their Treatment. 2008; 7:1-14.

Brewer JA, Worhunsky PD, Carroll KM, Rounsaville BJ, Potenza MN. Pretreatment brain activation during Stroop task is associated with outcomes in cocaine-dependent patients. Biological Psychiatry. 2008; 64:998-1004. [PubMed: 18635157] 
Brewer JA, Potenza MN. The Neurobiology and Genetics of Impulse Control Disorders: Relationships to Drug Addictions. Biochemical Pharmacology. 2008; 75:63-75. [PubMed: 17719013]

Brewer JA, Smith JT, Bowen S, Marlatt GA, Potenza MN. Applying mindfulness-based treatments to co-occurring depression and substance use disorders: What can we learn from the brain? Addiction. 2010; 105:1698-1706. [PubMed: 20331548]

Brewer JA, Worhunsky PD, Gray JR, Tang Y-Y, Weber J, Kober H. Meditation experience is associated with differences in default mode network activity and connectivity. Proceedings of the National Academy of Sciences of the United States of America. 2011; 108:20254-20259. [PubMed: 22114193]

Brezing CA, Derevensky JL, Potenza MN. Non-substance addictive behaviors in youth: pathological gambling and problematic internet use. Child and Adolescent Psychiatric Clinics of North America. 2010; 19:625-641. [PubMed: 20682225]

Carroll K, Rounsaville BJ, Nich C, Gordon LT, Wirtz PW, Gawin FH. One year follow-up of psychotherapy and pharmacotherapy for cocaine dependence: Delayed emergence of psychotherapy effects. Archives of General Psychiatry. 1994; 51:989-997. [PubMed: 7979888]

Carroll KM, Ball SA, Martino S, Nich C, Babuscio TA, Rounsaville BJ. Enduring effects of a computer-assisted training program for cognitive behavioral therapy: a 6-month follow-up of CBT4CBT. Drug and Alcohol Dependence. 2009; 100(1-2):178-181. doi: 10.1016/j.drugalcdep. 2008.09.015. [PubMed: 19041197]

Cavalca E, Kong G, Liss T, Reynolds EK, Schepis TS, Lejuez CW, Krishnan-Sarin S. A preliminary experimental investigation of peer influence on risk-taking among adolescent smokers and nonsmokers. Drug and Alcohol Dependence. 2012 doi: 10.1016/j.drugalcdep.2012.09.020.

Champine RB, Petry NM. Pathological gamblers respond equally well to cognitive-behavioral therapy regardless of other mental health treatment status. The American Journal on Addictions / American Academy of Psychiatrists in Alcoholism and Addictions. 2010; 19:550-556. [PubMed: 20958852]

Chase HW, Clark L. Gambling Severity Predicts Midbrain Response to Near-Miss Outcomes. The Journal of Neuroscience : the Official Journal of the Society for Neuroscience. 2010; 30:6180 6187. [PubMed: 20445043]

Chung SK, You IH, Cho GH, Chung GH, Shin YC, Kim DJ, Choi SW. Changes of functional MRI findings in a patient whose pathological gambling improved with fluvoxamine. Yonsei Medical Journal. 2009; 50(3):441-444. doi: 10.3349/ymj.2009.50.3.441. [PubMed: 19568609]

Clark L. Decision-making during gambling: an integration of cognitive and psychobiological approaches. Philosophical transactions of the Royal Society of London. Series B, Biological sciences. 2010; 365:319-330.

Clark L, Lawrence AJ, Astley-Jones F, Gray N. Gambling Near-Misses Enhance Motivation to Gamble and Recruit Win-Related Brain Circuitry. Neuron. 2010; 61:481-490. [PubMed: 19217383]

Crockford D, Goodyear B, Edwards J, Quickfall J, El-Guebaly N. Cue-induced brain activity in pathological gamblers. Biological Psychiatry. 2005; 58:787-795. [PubMed: 15993856]

Desai RA, Potenza MN. Gender differences in the associations between problem gambling and psychiatric disorders. Social Psychiatry and Psychiatric Epidemiology. 2008; 43:173-183. [PubMed: 18080792]

DeVito EE, Worhunsky PD, Carroll KM, Rounsaville BJ, Kober H, Potenza MN. A preliminary study of the neural effects of behavioral therapy for substance use disorders. Drug Alcohol Dependence. 2012; 122(3):228-235. doi: 10.1016/j.drugalcdep.2011.10.002.

Feldstein Ewing SF, Filbey FM, Hendershot CS, McEachern AD, Hutchison KE. Proposed model of the neurobiological mechanisms underlying psychosocial alcohol interventions: the example of motivational interviewing. Journal of Studies on Alcohol and Drugs. 2011; 72:903-916. [PubMed: 22051204]

Feldstein Ewing SF, Filbey FM, Sabbineni A, Chandler LD, Hutchison KE. How psychosocial alcohol interventions work: a preliminary look at what fRMI can tell us. Alcoholism, Clinical and Experimental Research. 2011; 35:643-651. 
Frascella J, Potenza MN, Brown LJ, Childress AR. Shared brain vulnerabilities open the way for nonsubstance addictions: carving addiction at a new joint? Annals of the New York Academy of Sciences. 2010; 1187:294-315. [PubMed: 20201859]

Gerstein, D.; Hoffmann, J.; Larison, C.; Engelman, L.; Murphy, S.; Palmer, A.; Hill, M. [March 27, 1999., 1999] Gambling impact and behavior study.. 1999.

Gooding P, Tarrier N. A systematic review and meta-analysis of cognitive-behavioural interventions to reduce problem gambling: hedging our bets? Behaviour Research and Therapy. 2009; 47(7):592607. doi: S0005-7967(09)00087-4 [pii]10.1016/j.brat.2009.04.002. [PubMed: 19446287]

Grant JE, Black DW, Stein DJ, Potenza MN. Clinical Case Discussion: Pathological Gambling and Nicotine Dependence. J Addict Med. 2009; 3:120-127. [PubMed: 20161572]

Grant, JE.; Donahue, CB.; Odlaug, BL. Overcoming Impulse Control Disorders: A CognitiveBehavioral Therapy Program (Workbook). Oxford University Press; New York, NY: 2011 a.

Grant, JE.; Donahue, CB.; Odlaug, BL. Treating Impulse Control Disorders: A Cognitive-Behavioral Therapy Program (Therapist Guide). Oxford University Press; New York, NY: 2011b.

Grant JE, Donahue CB, Odlaug BL, Kim SW. A 6-month follow-up of imaginal desensitization plus motivational interviewing in the treatment of pathological gambling. Annals of Clinical Psychiatry : Official Journal of the American Academy of Clinical Psychiatrists. 2011; 23:3-10. [PubMed: 21318190]

Grant JE, Donahue CB, Odlaug BL, Kim SW, Miller MJ, M. PN. Imaginal desensitization plus motivational interviewing for pathological gambling: a randomised controlled trial. The British Journal of Psychiatry: the Journal of Mental Science. 2009; 195:266-267. [PubMed: 19721120]

Habib R, Dixon MR. Neurobehavioral evidence for the "Near-Miss" effect in pathological gamblers. Journal of the Experimental Analysis of Behavior. 2010; 93(3):313-328. doi: 10.1901/jeab. 2010.93-313. [PubMed: 21119848]

Hampshire A, Owen AM. Fractionating attentional control using eventrelated fMRI. Cerebral Cortex. 2006; 16(12):1679-1689. doi: 10.1093/cercor/bhj116. [PubMed: 16436686]

Harsan LA, Steibel J, Zaremba A, Agin A, Sapin R, Poulet P. Recovery from chronic demyelination by thyroid hormone therapy: myelinogenesis induction and assessment by diffusion tensor magnetic resonance imaging. Journal of Neuroscience. 2008; 28:14189-14201. al, e. [PubMed: 19109501]

Hodgins DC, Peden N. Cognitive-behavioral treatment for impulse control disorders. Revista Brasileira de Psiquiatria. 2008; 30(Suppl 1):S31-S40. [PubMed: 17713695]

Holden C. 'Behavioral' addictions: Do they exist? Science. 2001; 294:980-982. [PubMed: 11691967]

Holden C. Behavioral addictions debut in proposed DSM-V. Science. 2010; 327:935. [PubMed: 20167757]

Hollander E, Pallanti S, Allen A, Sood E, Rossi NB. Does Sustained- Release Lithium Reduce Impulsive Gambling and Affective Instability Versus Placebo in Pathological Gamblers With Bipolar Spectrum Disorders? American Journal of Psychiatry. 2005; 162:137-145. [PubMed: 15625212]

Hu J, Henry S, Gallezot J-D, Ropchan J, Neumaier JF, Potenza MN, Neumeister A. Serotonin 1B Receptor Imaging in Alcohol Dependence. Biological Psychiatry. 2010; 63:300-303.

Hyatt CJ, Assaf M, Muska CE, Rosen RI, Thomas AD, Johnson MR, Pearlson GD. Reward-related dorsal striatal activity differences between former and current cocaine dependent individuals during an interactive competitive game. PloS One. 2012; 7(5):e34917. doi: 10.1371/journal.pone. 0034917 PONE-D-11-17628 [pii]. [PubMed: 22606228]

Joutsa J, Saunavaara J, Parkkola R, Niemela S, Kaasinen V. Extensive abnormality of brain white matter integrity in pathological gambling. Psychiatry Research. 2011; 194(3):340-346. doi: 10.1016/j.pscychresns.2011.08.001. [PubMed: 22079653]

Knutson B, Fong GW, Bennett SM, Adams CM, Hommer D. A region of mesial prefrontal cortex tracks monetarily rewarding outcomes: characterization with rapid event-related fMRI. Neuroimage. 2003; 18:263-272. [PubMed: 12595181]

Kober H, Mende-Siedlecki P, Kross EF, Weber J, Mischel W, Hart CL, Ochsner KN. Prefrontalstriatal pathway underlies cognitive regulation of craving. Proceedings of the National Academy of Sciences of the United States of America. 2010; 107(33):14811-14816. [PubMed: 20679212]

Psychol Addict Behav. Author manuscript; available in PMC 2014 June 01. 
Ladouceur, R.; Lachance, S. Overcoming pathological gambling: Therapist guide. Oxford University Press; New York, NY: 2007a.

Ladouceur, R.; Lachance, S. Overcoming your pathological gambling: workbook. Oxford University Press; New York, NY: 2007b.

Lawrence AJ, Luty J, Bogdan NA, Sahakian BJ, Clark L. Problem gamblers share deficits in impulsive decision-making with alcohol dependent individuals. Addiction. 2009; 104:1006-1015. [PubMed: 19466924]

Leeman RF, Grant JE, Potenza MN. Behavioral and neurological foundations for the moral and legal implications of intoxication, addictive behaviors and disinhibition. Behavioral Sciences and the Law. 2009; 27:237-259. [PubMed: 19241397]

Leeman RF, Potenza MN. Similarities and Differences between Pathological Gambling and Substance Use Disorders: A Focus on Impulsivity and Compulsivity. Psychopharmacology. 2012; 219:469_ 490. [PubMed: 22057662]

McClure SM, Laibson DI, Loewenstein G, Cohen JD. Separate neural systems value immediate and delayed monetary rewards. Science. 2004; 306:503-507. [PubMed: 15486304]

McConaghy N, Blaszczynski A, Frankova A. Comparison of imaginal desensitisation with other behavioural treatments of pathological gambling. A two- to nine-year follow-up. British Journal of Psychiatry. 1991; 159:390-393. [PubMed: 1683593]

Miedl SF, Peters J, Buchel C. Altered Neural Reward Representations in Pathological Gamblers Revealed by Delay and Probability Discounting. Archives of General Psychiatry. 2012; 69:177186. [PubMed: 22310505]

Miller WR. Motivational interviewing: research, practice, and puzzles. Addictive Behaviors. 1996; 21:835-842. [PubMed: 8904947]

Miller WR, Rollnick S. Ten things that motivational interviewing is not. Behavioural and Cognitive Psychotherapy. 2009; 37:129-140. [PubMed: 19364414]

Miller WR, Yahne CE, Tonigan JS. Motivational interviewing in drug abuse services: a randomized trial. Journal of Consulting and Clinical Psychology. 2003; 71:754-763. [PubMed: 12924680]

Pallanti S, DeCaria CM, Grant JE, Urpe M, Hollander E. Reliability and validity of the pathological gambling adaptation of the Yale-Brown Obsessive-Compulsive Scale (PG-YBOCS). Journal of Gambling Studies. 2005; 21:431-443. [PubMed: 16311876]

Pallanti S, Haznedar MM, Hollander E, Licalzi EM, Bernardi S, Newmark R, Buchsbaum MS. Basal Ganglia activity in pathological gambling: a fluorodeoxyglucose-positron emission tomography study. Neuropsychobiology. 2010; 62:132-138. [PubMed: 20588072]

Pallesen S, Mitsem M, Kvale G, Johnsen BH, Molde H. Outcome of psychological treatments of pathological gambling: a review and meta-analysis. Addiction. 2005; 100:1412-1422. [PubMed: 16185203]

Petry NM. Patterns and correlates of gamblers anonymous attendance in pathological gamblers seeking professional treatment. Addictive Behaviors. 2003; 28:1049-1062. [PubMed: 12834650]

Petry NM. Gamblers Anonymous and Cognitive-Behavioral Therapies for Pathological Gamblers. Journal of Gambling Studies. 2005a; 21:27-33. [PubMed: 15789187]

Petry, NM. Pathological gambling: Etiology, comorbidity and treatment. American Psychological Association; Washington, DC: 2005b.

Petry NM, Stinson FS, Grant BF. Co-morbidity of DSM-IV pathological gambling and other psychiatric disorders: Results from the National Epidemiologic Survey on Alcohol and Related Conditions. Journal of Clinical Psychiatry. 2005; 66:564-574. [PubMed: 15889941]

Petry NM, Weinstock J, Ledgerwood DM, Morasco B. A randomized trial of brief interventions for problem and pathological gamblers. Journal of Consulting and Clinical Psychology. 2008; 76:318328. [PubMed: 18377127]

Potenza MN. Should addictive disorders include non-substance-related conditions? Addiction. 2006; 101(s1):142-151. [PubMed: 16930171]

Potenza MN. The neurobiology of pathological gambling and drug addiction: an overview and new findings. Philosophical Transactions of the Royal Society of London. Series B, Biological sciences. 2008; 363:3181-3189. 
Potenza MN. Non-substance and substance addictions. Addiction. 2009; 104:1016-1017. [PubMed: 19466925]

Potenza MN, Hong KI, Lacadie CM, Fulbright RK, Tuit KL, Sinha R. Neural correlates of stressinduced and cue-induced drug craving: influences of sex and cocaine dependence. American Journal of Psychiatry. 2012; 169(4):406-414. doi: 10.1176/appi.ajp.2011.11020289. [PubMed: 22294257]

Potenza MN, Kosten TR, Rounsaville BJ. Pathological gambling. JAMA : the Journal of the American Medical Association. 2001; 286:141-144. [PubMed: 11448261]

Potenza MN, Leung H-C, Blumberg HP, Peterson BS, Skudlarski P, Lacadie C, Gore JC. An fMRI stroop study of ventromedial prefrontal cortical function in pathological gamblers. American Journal of Psychiatry. 2003; 160:1990-1994. [PubMed: 14594746]

Potenza MN, Sofuoglu M, Carroll KM, Rounsaville BJ. Neuroscience of behavioral and pharmacological treatments for addictions. Neuron. 2011; 69:695-712. [PubMed: 21338880]

Potenza MN, Steinberg MA, Skudlarski P, Fulbright RK, Lacadie C, Wilber MK, Wexler BE. Gambling urges in pathological gambling. Archives of General Psychiatry. 2003; 60:828-836. [PubMed: 12912766]

Potenza MN, Walderhaug E, Henry S, Gallezot J, Planeta-Wilson B, Ropchan J, Neumeister A. Serotonin 1B receptor imaging in pathological gambling. World Journal of Biological Psychiatry. in press.

Rao H, Mamikonyan E, Detre JA, Siderowf AD, Stern MB, Potenza MN, Weintraub D. Decreased ventral striatal activity with impulse control disorders in Parkinson's disease. Movement Disorders. 2010; 25(11):1660-1669. doi: 10.1002/mds.23147. [PubMed: 20589879]

Reuter J, Raedler T, Rose M, Hand I, Glascher J, Buchel C. Pathological gambling is linked to reduced activation of the mesolimbic reward system. Nature Neuroscience. 2005; 8:147-148.

Rogers RD, Andrews TC, Grasby PM, Brooks DJ, Robbins TW. Contrasting cortical and subcortical activations produced by attentional-set shifting and reversal learning in humans. Journal of Cognitive Neuroscience. 2000; 12(1):142-162. [PubMed: 10769312]

Schlaug G, Marchina S, Norton A. Evidence for plasticity in white-matter tracts of patients with chronic Broca's aphasia undergoing intense intonation-based speech therapy. Annals of the New York Academy of Sciences. 2009; 1169:385-394. [PubMed: 19673813]

Shaffer H, Hall MN, Vander Bilt J. Estimating the prevalence of disordered gambling in the United States and Canada: A research synthesis. American Journal of Public Health. 1999; 89:1369-1376. [PubMed: 10474555]

Slutske WS, Eisen S, True WR, Lyons MJ, Goldberg J, Tsuang M. Common genetic vulnerability for pathological gambling and alcohol dependence in men. Archives of General Psychiatry. 2000; 57:666-674. [PubMed: 10891037]

Stea JN, Hodgins DC. A critical review of treatment approaches for gambling disorders. Current Drug Abuse Reviews. 2011; 4:67-80. [PubMed: 21696346]

Steeves TDL, Miyasaki J, Zurowski M, Lang AE, Pellecchia G, van Eimeren T, Strafella AP. Increased striatal dopamine release in Parkinsonian patients with pathological gambling: a [11C] raclopride PET study. Brain. 2009; 132:1376-1385. [PubMed: 19346328]

Stewart RM, Brown RI. An outcome study of Gamblers Anonymous. British Journal of Psychiatry. 1988; 152:284-288. [PubMed: 3167353]

Streeter C, Terhune D, Whitfield T, Gruber S, Sarid-Segal O, Silveri M, Yurgelin-Todd D. Performance on the Stroop predicts treatment compliance in cocaine-dependent individuals. Neuropsychopharmacology. 2007; 33:827-836. [PubMed: 17568399]

Sylvain C, Ladouceur R, Boisvert J-M. Cognitive and behavioral treatment of pathological gambling: A controlled study. Journal of Consulting and Clinical Psychology. 1997; 65(5):727-732. [PubMed: 9337491]

Tanabe J, Thompson L, Claus E, Dalwani M, Hutchison K, Banich MT. Prefrontal Cortex Activity is Reduced in Gambling and Nongambling Substance Users During Decision-Making. Human Brain Mapping. 2007; 28:1276-1286. [PubMed: 17274020] 
Tang Y, Lu Q, Geng X, Stein EA, Yang Y, Posner M. Short-term meditation induces white matter changes in the anterior cingulate. Proceedings of the National Academy of Sciences of the United States of America. 2010; 107:15649-15652. [PubMed: 20713717]

Tavares H, Zilberman ML, el-Guebaly N. Are there cognitive and behavioural approaches specific to the treatment of pathological gambling? Canadian Journal of Psychiatry. 2003; 48:22-27.

Toneatto T, Brands B, Selby P. A randomized, double-blind, placebo-controlled trial of naltrexone in the treatment of concurrent alcohol use disorder and pathological gambling. The American Journal on Addictions / American Academy of Psychiatrists in Alcoholism and Addictions. 2009; 18:219225. [PubMed: 19340640]

Worhunsky PD, Calhoun VD, Stevens M, Pearlson GD, Carroll KM, Rounsaville BJ, Potenza MN. Independent component analysis of fMRI Stroop in cocaine dependent subjects: Relationship to treatment outcome. Psychology of Addictive Behaviors : Journal of the Society of Psychologists in Addictive Behaviors. in press.

Wrase J, Schlagenhauf F, Kienast T, Wüstenberg T, Bermpohl F, Kahnt T, Heinz A. Dysfunction of reward processing correlates with alcohol craving in detoxified alcoholics. Neuroimage. 2007; 35:787-794. [PubMed: 17291784]

Xu J, DeVito EE, Worhunsky PD, Carroll KM, Rounsaville BJ, Potenza MN. White Matter Integrity is Associated with Treatment Outcome Measures in Cocaine Dependence. Neuropsychopharmacology. 2010; 35:1541-1549. [PubMed: 20393459]

Yin HH, Knowlton BJ. The role of the basal ganglia in habit formation. Nature Reviews Neuroscience. 2006; 7(6):464-476.

Yip SW, Lacadie C, Xu J, Worhunsky PD, Fulbright RK, Constable RT, Potenza MN. Reduced genual corpus collosal white matter integrity in pathological gambling and its relationship to alcohol abuse or dependence. World Journal of Biological Psychiatry. in press. 


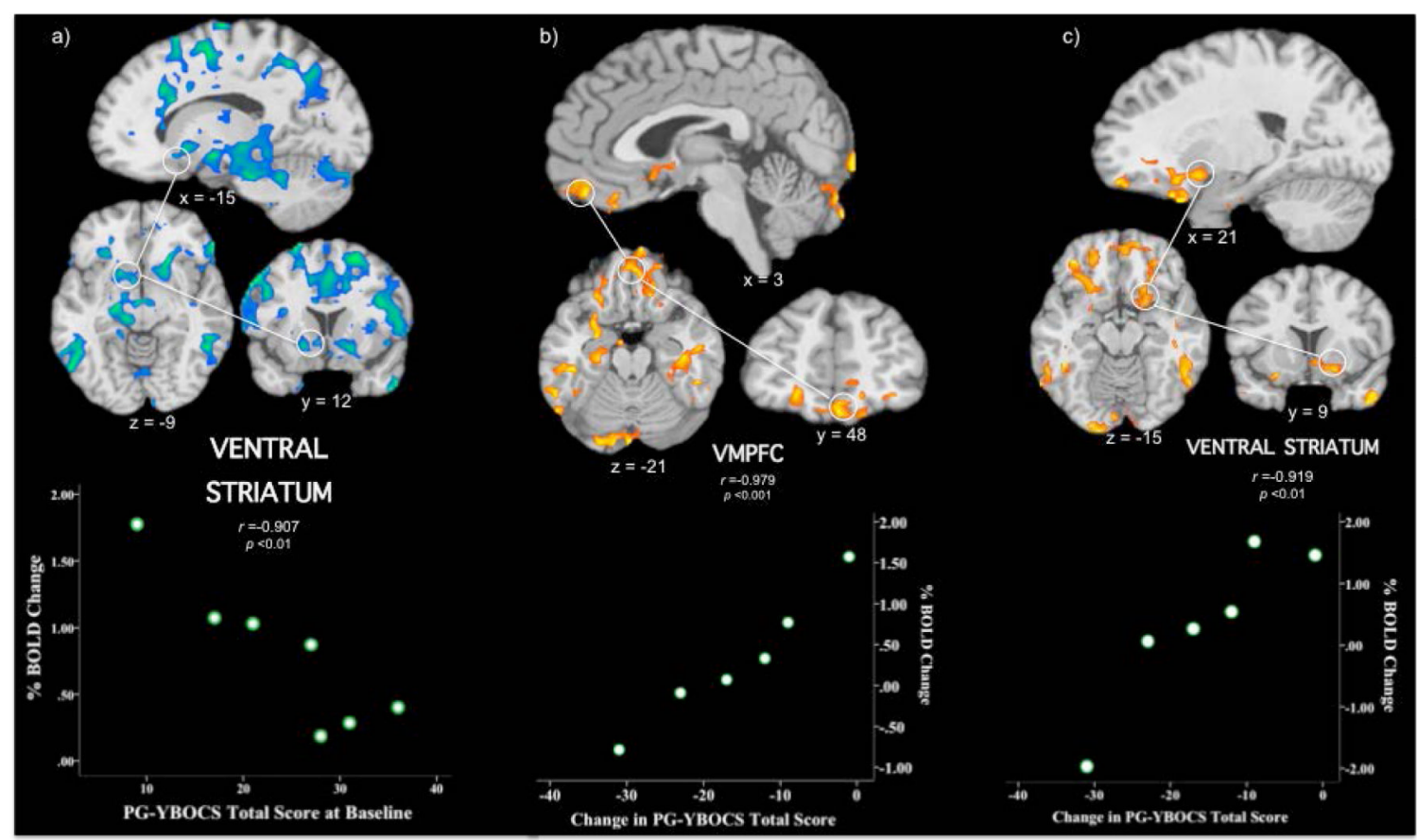

Figure 1. Saggital, axial and coronal views of regional brain activation during Stroop task performance and correlations with PG-YBOCS scores

a) At baseline, brain slices highlight the correlation with a cluster in the left ventral striatum $(-15,12,-9 ; n=7$ subjects); b) Absolute changes in PG-YBOCS scores (end of treatment vs pre-treatment) correlate with a cluster in the $\operatorname{vmPFC}(3,48,-21 ; n=6$ subjects); c) Absolute changes in PG-YBOCS scores (end of treatment vs pre-treatment) correlate with a cluster in the ventral striatum $(21,9,-15 ; n=6$ subjects). Orange/yellow indicates areas of positive correlations and blue/green indicates areas of negative correlations between PG-YBOCS scores and \% BOLD signal changes in the incongruent versus congruent contrast. All maps are thresholded at an uncorrected level of $p<0.05$ and two-tailed FWE-corrected at $p<0.05$ with a cluster threshold of 90 . Right side of the brain is on the right. Scatterplots (bottom of figure) demonstrate the distribution of individual scores in the correlation between absolute changes in PG-YBOCS scores and \% BOLD signal change during incongruent versus congruent stimuli presentation during Stroop task performance. BOLD = blood oxygenation level-dependent; FWE = family-wise error; MNI = Montreal Neurological Institute; PGYBOCS = Yale-Brown Obsessive Compulsive Scale modified for Pathological Gambling; $r$ $=$ Pearson correlation coefficient; $\mathrm{vmPFC}=$ ventral medial prefrontal cortex 


\begin{tabular}{|c|c|c|c|c|c|c|c|c|c|}
\hline 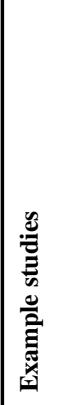 & 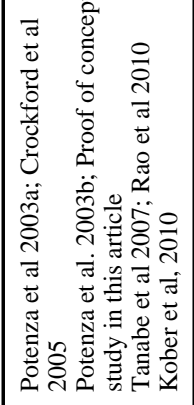 & 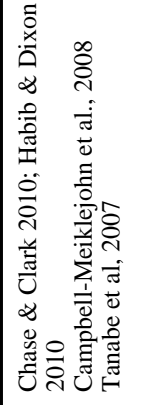 & 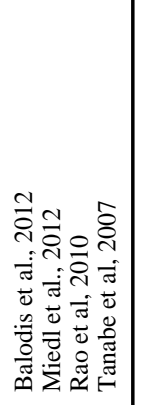 & 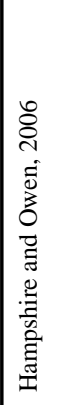 & 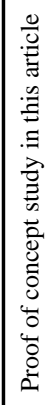 & 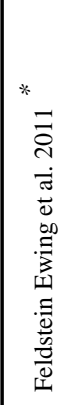 & 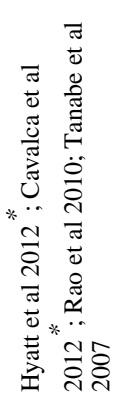 & 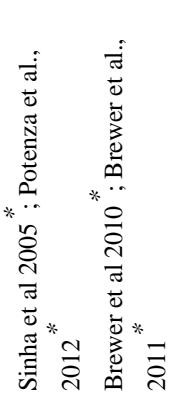 & 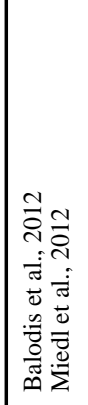 \\
\hline & 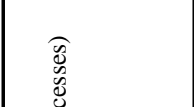 & . & & & & $\frac{\partial}{5}$ & $\stackrel{\text { on }}{\stackrel{0}{2}}$ & $\frac{1}{5}$ & \\
\hline
\end{tabular}

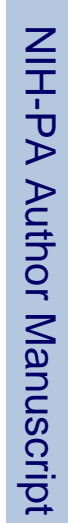

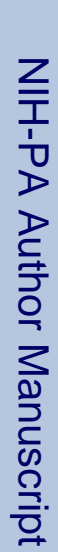

$\frac{\overline{0}}{\frac{0}{\circ}}$

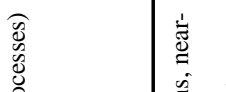
施
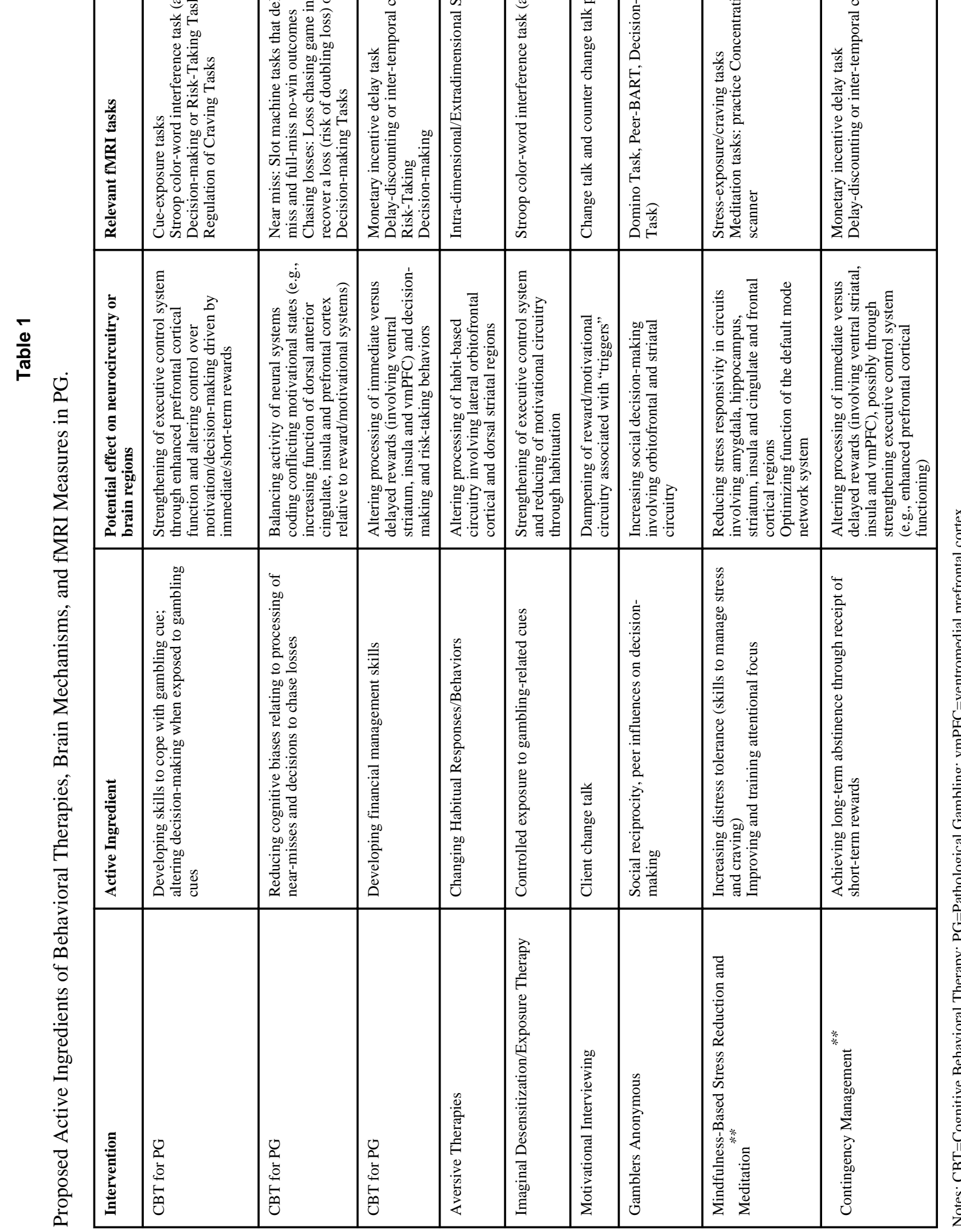
